\title{
15-LOX Inhibitors: Biochemical Evaluation of Flurbiprofen and its Derivatives
}

Saghir Abbas ${ }^{1}$, Sumera Zaib², Saqib Ali $^{3}$, Jamshed Iqbal ${ }^{2}$

\section{ABSTRACT}

Objective: The synthesis, characterization, 15-LOX inhibition and molecular docking studies of a commercially available NSAID, flurbiprofen and its derivatives.

Study Design: Experimental study.

Place and Duration of Study: The study was carried out at Quaid-i-Azam University and Centre for Advanced Drug Research COMSATS University Islamabad, Abottabad Campus from March 2019 to February 2020.

Materials and Methods: The structural elucidation of the compounds (2-5) was carried out using infrared, ${ }^{1} \mathrm{H}$ and ${ }^{13} \mathrm{C}$ NMR spectroscopic studies. The structure of 4-amino-5-(1-(2-fluorobiphenyl-4-yl)-ethyl)-4H-1,2,4triazole-3-thione (5) was also verified by single crystal X-ray diffraction (XRD) studies.

Results: The most potent inhibitor for 15-LOX (2) has an IC $\mathrm{C}_{50}$ value of $0.18 \pm 0.01 \mu \mathrm{M}$. Molecular docking results of 1, 2, 3 and cognate ligand inside the active site of 15-LOX (PDB ID: 1IK3) revealed significant correlation.

Conclusion: This work represents cost-effective, reproducible and facile conversion of an aromatic monocarboxylic acid into potent derivatives.The molecules 2-(2-fluorobiphenyl-4-yl) propanoic acid (1) and its derivatives (2-5) possess 15-LOX inhibition and can be a prospective therapeutic target for chronic obstructive pulmonary disease.

Key Words: Hydrazide, Lipoxygenase Inhibition, Molecular Docking, X-Ray Diffraction, 1,2,4-Triazole-3-Thione.

How to cite this: Abbas S, Zaib S, Ali S, Iqbal J. 15-LOX Inhibitors: Biochemical Evaluation of Flurbiprofen and its Derivatives. Life and Science. 2020; 1(3): 92-97. doi: http://doi.org/10.37185/LnS.1.1.107

This is an Open Access article distributed under the terms of the Creative Commons Attribution License (http://creativecommons.org/licenses/by/4.0), which permits unrestricted use, distribution, and reproduction in any medium, provided the original work is properly cited.

\section{Introduction}

Lipoxygenase (LOX) join a non-heme family of iron containing dioxygenase enzymes which introduce oxygen into linoleic acid and arachidonic acid through regio- and stereospecific pathway and convert these fatty acids into hydroperoxyl fatty acids. ${ }^{1-3}$ These hydroperoxy fatty acids are converted into leukotrienes and lipoxins which are important regulators for many diseases. The LOX family contains 5-LOX, 9-LOX, 12-LOX, and 15-LOX isozymes,

\footnotetext{
${ }^{1}$ Department of Biological Sciences

National University of Medical Sciences, Rawalpindi

${ }^{2}$ Centre for Advanced Drug Research

COMSATS University

Abbottabad Campus, Abbottabad

${ }^{3}$ Department of Chemistry

Quaid-I-Azam University, Islamabad

Correspondence:

Prof Dr. Jamshed lqbal

Centre for Advanced Drug Research

COMSATS University

Abbottabad Campus, Abbottabad

E-mail:drjamshed@cuiatd.edu.pk

Funding Source: HEC; Conflict of Interest: NIL

Received: Mar 24, 2020; Revised: Apr 21, 2020

Accepted: Jun 08, 2020
}

which-oxidize the main fatty acid arachidonic acid at their respective 5, 9, 12 and 15 positions. ${ }^{4}$ These isozymes also perform crucial part in the development of the pathophysiology of many diseases in humans, thus they could be a potential targets for $f$ therapeutic regime modulation. ${ }^{5}$ Among them 15-LOX is the most influential target, which is involved in pathology of certain carcinomas, chronic obstructive pulmonary disease (COPD) and atherosclerosis. $^{6-12}$

15-LOX has this name because it catalyses addition of oxygen at position 15 of arachidonic acid. The oxidation of linoleic acid with LOX isozymes can produce pro-thrombotic ${ }^{13}$, pro-inflammatory ${ }^{14}$ and leukotrienes molecules. ${ }^{15}$ Leukotrienes are highly involved in multiple pathological conditions, i.e., asthma, pleural effusion, pneumonia, tuberculosis, pulmonary embolism, congestive heart failure and lung cancer etc. ${ }^{16-18}$ Thus, inhibition of lipoxygenases prevents development of leukotrienes and related pro-inflammatory metabolite production. A significant number of lipoxygenase inhibitors have reached $\log P$ values of 5 and above, but the 
determined lipid ligand performances are generally low. From this viewpoint, it is extremely important to discover new analogues for innovative substitution patterns for formerly known lipoxygenase binding core structures. This mechanism can introduce inhibitors with improved physicochemical characteristics.

Heterocyclic triazole compounds have tremendous applications as synthetic intermediates in medicines $^{19}$ as antimicrobial ${ }^{20,21}$, antibacterial ${ }^{22}$, and antitrypanosomal agents. ${ }^{23}$ Triazole is a $\pi$-conjugated system carrying $\mathrm{sp}^{3}$-amine and $\mathrm{sp}^{2}$-imine-type nitrogen atoms with multiple coordination modes rendering them superior to imidazole and pyrazoles. Triazoles are generally classified as bases because they happen in the protonated form as azolium cation, however they can also be deprotonated to form azolate (or azolide) anion. Triazole bases can be synthesized by the modification of carboxylic functional group of nonsteroidal anti-inflammatory drugs (NSAIDs) for potential anti-viral and in vitro cytotoxic applications. ${ }^{24-26}$

In the current study, we present the synthesis, characterization, 15-LOX inhibition and molecular docking studies of a commercially available (NSAID) flurbiprofen and its derivatives.

\section{Materials and Methods}

2-(2-fluorobiphenyl-4-yl) propanoic acid (1) and the solvents used were bought from E. Merck (Germany). The solvents were dried before use employing the standard procedures. ${ }^{27}$ The biological test materials were obtained from as mentioned previously. ${ }^{28}$ A Gallenkamp melting point apparatus was utilized to determine melting points. Infrared measurements were recorded on a Bruker Tensor II spectrophotometer. ${ }^{1} \mathrm{H}$ and ${ }^{13} \mathrm{C}-\mathrm{NMR}$ spectra were determined on a Bruker $300 \mathrm{MHz}$ NMR spectrometer using deuterated dimethyl sulphoxide. Single crystal XRD studies were done using a Bruker Kappa APEX-II CCD diffractometer (Mo X-ray source). SHELXL-97 was used to obtain final refinements of the crystal structure. ${ }^{29,30}$

Chemical synthesis: The compounds (2-5) were synthesized adopting the same synthesis route reported in our previous work. ${ }^{28}$ All the derivatives were obtained in good yield (70-80 \%). Procedure for the synthesis of 2 and 3: A condensation reaction was carried out in order to synthesize ethyl-2-(2-fluorobiphenyl-4-yl) propanoate (2). To an ethanolic solution of 2-(2fluorobiphenyl-4-yl) propanoic acid $(2 \mathrm{mmol})$ few drops of conc. $\mathrm{H}_{2} \mathrm{SO}_{4}$ catalyst was added and the resulting solution was kept at reflux conditions for 6 hours. Formation of the product was checked using pre-coated silica gel TLC plates applying chloroform: methanol (8:2) eluent. The reaction mixture was rotary evaporated to extract ester from alcohol and was purified by solvent extraction methodology using a $\mathrm{NaHCO}_{3}$ solution. The obtained ester was co nverted into 2-(2-fluorobiphenyl-4-yl) propane hydrazide (3) by dissolving compound 2 and hydrazine hydrate $(1: 2)$ in $\mathrm{C}_{2} \mathrm{H}_{5} \mathrm{OH}(50 \mathrm{~mL})$ and refluxing the reaction mixture for $8 \mathrm{hrs}$ (scheme $\mathrm{A}$ ). TLC plates were used to monitor the reaction progress employing petroleum ether: ethyl acetate (1:1) eluent. A pure yellow colour solid of hydrazide (3) was achieved by recrystallization from methanol. Procedure for the synthesis of 4 and 5: To an icecooled alcoholic $\mathrm{KOH}$ (0.03 moles) solution, 2-(2fluorobiphenyl-4-yl) propane hydrazide (0.015 moles) and carbon disulphide $(0.025 \mathrm{~mol})$ was added. The reaction progressed at reflux conditions and constant stirring for 12 hours. A solid potassium thiocarbamate (4) was obtained by rotary evaporation of solution. Potassium thiocarbamate was washed several times with ethanol, dried and employed further for the ring closure reaction leading towards synthesis of a triazole moiety (5). For the preparation of a triazole compound, hydrazine hydrate $(0.02 \mathrm{~mol})$ was added dropwise to an aqueous solution of 4 . The resulting solution was kept at reflux conditions for 10 hours where a release of the hydrogen sulphide gas was observed during reaction turning the reaction mixture green. The reaction mixture was cooled and acidified using dilute $\mathrm{HCl}$ to produce the desired product (5). At this time, toluene: ethyl acetate: formic acid (5:4:1) eluent was employed for TLC monitoring of the reaction.

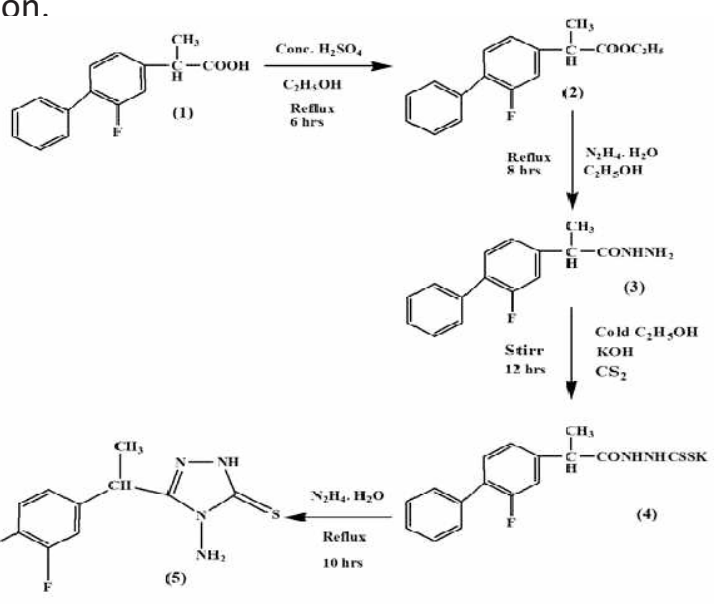

Scheme A: Synthesis of 4-amino-5-(1-(2-fluorobiphenyl4-yl)-ethyl)-4H-1,2,4-triazole-3-thione (5). 
Physical measurements: The physical measurements have been reported in our previous work. ${ }^{28}$

\section{Biological assays}

15-Lipoxygenase inhibition assay: Lipoxygenase inhibition studies were performed with slight modifications in previously reported spectrophotometric method. ${ }^{31}$ Briefly, $10 \mathrm{uL}$ of 15LOX (soybean source, $42.5 \mathrm{U}$ ) was mixed with $20 \mathrm{uL}$ compound ( $0.1 \mathrm{mM}$ in $1 \% \mathrm{DMSO}$ ) in $145 \mathrm{uL}$ of $\mathrm{KH}_{2} \mathrm{PO}_{4}$ buffer (100 mM, pH 8.0). The reaction mixture was incubated at $25^{\circ} \mathrm{C}$ for $10 \mathrm{~min}$ and absorbance was measured at $234 \mathrm{~nm}$ using a microplate reader (FLUOstar Omega (BMG Labtech, Offenburg, Germany). The reaction started with the addition of $25 \mathrm{uL}$ of linoleic acid as substrate, followed by incubation for $6 \mathrm{~min}$ and the change in absorbance was measured. Quercetin was used as a positive control. All the experiments were performed in triplicates. The compounds exhibiting $>50 \%$ inhibition was subjected to further evaluation of IC50 values by non-linear regression analysis software PRISM 5 (GraphPad, San Diego, California, USA).

Molecular docking: To evaluate the binding mode of dual inhibitors within the active site of the enzyme, high-resolution crystal structure of 15-LOX (PDB ID: 1IK3, $2.0 \AA$ resolution) was downloaded from the RCSB Protein Data Bank. To explore the binding mode of favourable inhibitors within the active site of enzyme different conformations of same ligands were studied. The enzyme structure was clarified for missing amino acids and bonds contacts. The receptors were prepared using $\mathrm{MOE}^{32}$ Docking studies of selected inhibitors and cognate ligand was carried out using LeadIT software using default parameters. ${ }^{33}$ The ligands were kept flexible with fixed protein conformation, docking was carried out treating all the single bonds as rotatable. Different conformations were obtained after each run and the top ranked poses with maximum cluster at minimum energies was selected for visualization using BIOVIA Discovery Sudio. ${ }^{34,35}$

\section{Results}

Spectroscopic study: The structural elucidation of 25 was carried out by IR, ${ }^{1} \mathrm{H}$ and ${ }^{13} \mathrm{C}$ NMR spectroscopy. The infrared spectrum showed a relatively strong absorption band at $3405 \mathrm{~cm}^{-1}$ and $3388 \mathrm{~cm}^{-1}$ for $\mathrm{VNH}_{2}$ and at 3196 and $3188 \mathrm{~cm}^{-1}$ for $v \mathrm{NH}$ group of hydrazide (3) and 1,2,4-triazole-thione (5) structure, respectively, confirming the formulation of products. The typical IR band at $1077 \mathrm{~cm}^{-1}$ for C-S stretching proved the existence of a thione moiety in 5 . The ${ }^{1} \mathrm{H}$ and ${ }^{13} \mathrm{C}$ NMR spectra for the flurbiprofen derivatives were obtained in deuterated solvents $\left(2,3\right.$ in $\mathrm{CDCl}_{3} ; 5$ in DMSO- $\mathrm{d}_{6}$ ). The chemical shift values assigned to protons and carbons harmonized well with the assessed molecular composition of $2-5 .{ }^{36}$ The ${ }^{1} \mathrm{H}$ and ${ }^{13} \mathrm{C}$ NMR spectra of 5 have been shown in Figure 1 and 2. The formation of 2-(2-fluorobiphenyl-4-yl) propanate (2) was inveterated due the absence of chemical shift value for the carboxylic proton and presence of a new peak at $4.21 \mathrm{ppm}$ for $-\mathrm{O}-\mathrm{CH}_{2}$ group in the ${ }^{1} \mathrm{H}$ NMR spectrum. The ${ }^{1} \mathrm{H}$ NMR spectrum exhibited a singlet at $8.20 \mathrm{ppm}$ for $\mathrm{NH}$ proton of 3 present contiguous to the $\mathrm{C}=\mathrm{O}$ group. Additionally, $\mathrm{a}$ broad singlet for two protons of $\mathrm{NH}_{2}$ group, at 3.60 ppm specified the preparation of 3 . A downfield shift for $\mathrm{NH}$ proton at $13.65 \mathrm{ppm}$ for the triazole moiety (5) matches well with the reported value. ${ }^{28}$ The NH proton in 5 occurs as thione-thiol tautomeric arrangement. The thione form dominates in the structure which was confirmed by the absence of absorption frequency for a thiol (-SH) functional group in the infrared spectrum and it was also supported by single crystal XRD data (Figure 1). The ${ }^{1} \mathrm{H}$ NMR presented a singlet at 5.50 for the $\mathrm{NH}_{2}$ group in $5 .{ }^{13} \mathrm{C}$ NMR spectroscopic results indicate the vanishing of $\{\mathrm{C}=\mathrm{O}\}$ peak of hydrazide (3) appeared at $173.4 \mathrm{ppm}$ while the appearance of a new peak for $\{\mathrm{C}=\mathrm{S}\}$ at $166.8 \mathrm{ppm}$ for a thione functional group indicated the synthesis of a 1,2,4-triazole-3-thione moiety.

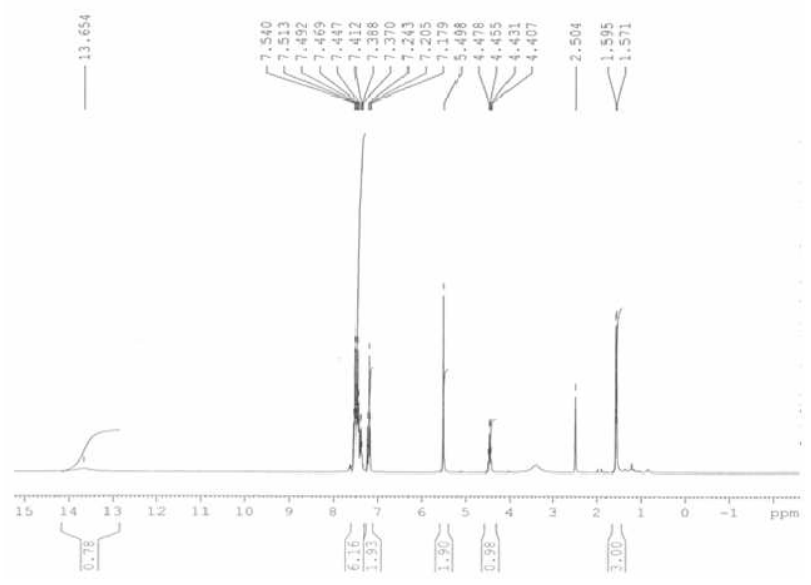

Fig 1. ${ }^{1} \mathrm{H}$ NMR spectrum of 5

X-ray diffraction studies: XRD analysis data has been previously reported by our group. ${ }^{28}$ The crystallographic information is provided in Table 1 


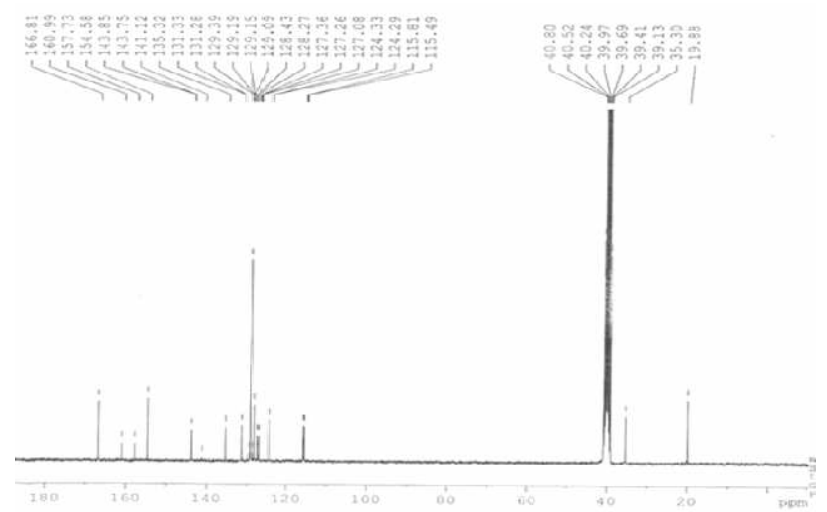

Fig 2: ${ }^{13} \mathrm{C}$ NMR spectrum of 5

while the hydrogen bond geometry in 5 is presented in Table 2. The molecular structure of 5 is given in Figure 3 while the symmetrical order and $\mathrm{H}$ interaction pattern is presented in Figure 4 and 5. Xray diffraction studies indicate that 5 crystallized out in a monoclinic crystal system with a space group $P 2_{1} / n$. Two $N-H$...S interactions occur, one of them ends into an $S(5)$ ring and other gives a dimer with $R_{2}^{2}$ (10) ring (Figure 4).

\begin{tabular}{ll}
\hline $\begin{array}{l}\text { Table 1: The crystal refinements and structural parameters } \\
\text { for } \mathbf{5}\end{array}$ & $\mathbf{5}$ \\
\hline Compound Properties & 1535916 \\
CCDC \# & $\mathrm{C}_{16} \mathrm{H}_{15} \mathrm{~F} \mathrm{~N}_{4} \mathrm{~S}$ \\
Chemical formula & 314.38 \\
Formula weight & Colourless \\
Crystal colour & Rod \\
Crystal description & $296(2)$ \\
$T / \mathrm{K}$ & 0.71073 \\
Wavelength / & Monoclinic \\
Crystal system & $\mathrm{P} 21 / \mathrm{n}$ \\
Space group & $(6.1601(2)$ \\
$a / \AA$ & $(13.7424(7))$ \\
$b / \AA$ & $18.1717(9)$ \\
$c / \AA$ & 90 \\
$\alpha /^{\circ}$ & $97.771(2)$ \\
$B /^{\circ}$ & 90 \\
$V /{ }^{\circ}$ & $1524.19(12)$ \\
$V / \AA^{3}$ & 4 \\
$Z$ & 656 \\
$\mathrm{~F}(000)$ & \\
\hline
\end{tabular}

Table 2: Hydrogen -bond geometry (Å) in compound 5

\begin{tabular}{llllc}
\hline $\mathrm{D}-\mathrm{H} \cdots \mathrm{A}$ & $\mathrm{D}-\mathrm{H}$ & $\mathrm{H} \cdots \mathrm{A}$ & $\mathrm{D} \cdots \mathrm{A}$ & $\mathrm{D}-\mathrm{H} \cdots \mathrm{A}$ \\
$\mathrm{O} 2-\mathrm{H} 2 A \cdots \mathrm{N} 2$ & 0.82 & 1.93 & $2.650(3)$ & 146 \\
$\mathrm{~N} 1-\mathrm{H} 1 \cdots \mathrm{O} 3^{\mathrm{i}}$ & 0.86 & 2.01 & $2.858(3)$ & 170 \\
$\mathrm{O} 4-\mathrm{H} 4 A \cdots \mathrm{N} 4$ & 0.82 & 1.89 & $2.615(3)$ & 147 \\
$\mathrm{~N} 3-\mathrm{H} 3 A \cdots \mathrm{O} 1^{\mathrm{ii}}$ & 0.86 & 2.17 & $2.846(3)$ & 135 \\
\hline
\end{tabular}

Symmetry codes: (i) $-x+1 / 2, y+1 / 2, z-1 / 2$

(ii) $-x+1 / 2, y-1 / 2, z-1 / 2$

95

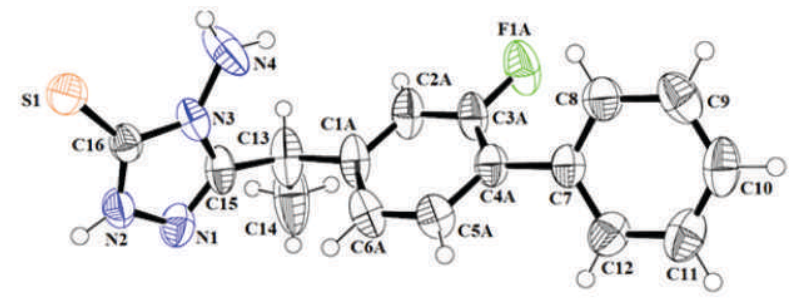

Fig 3: ORTEP diagram of 5 with atomic numbering scheme

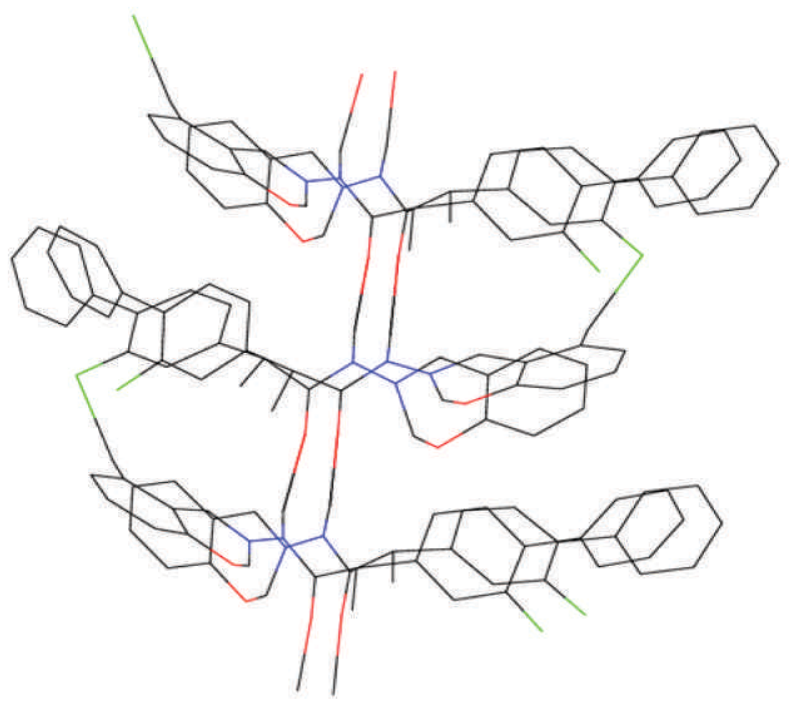

Fig 4: The symmetrical order of the molecule 5

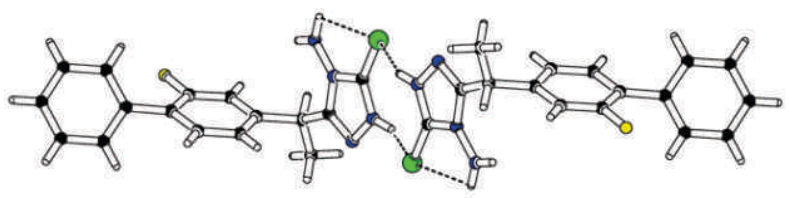

Fig 5: $\mathrm{H}$-interactions in 5

15-Lipoxygenase inhibition assay: 15-Lipoxygenase inhibition assay was performed on all the synthesized compounds (2-5) and their precursor (1) (Table 3). The ester derivative (2) of flurbiprofen showed promising results against 15-LOX enzyme with an $\mathrm{IC}_{50}$ value of $0.18 \pm 0.01 \mu \mathrm{M}$ only depicting an 87-fold higher inhibition than the reference quercetin (positive control). Moreover, compounds 1 and 3 also exhibited very promising inhibition results against 15-LOX.

\begin{tabular}{cc}
\hline $\begin{array}{c}\text { Table 3: 15-Lipoxygenase inhibition results against } \\
\text { flurbiprofen (1) and its derivatives }\end{array}$ \\
\hline Codes & 15-LOX $_{\left(\mathrm{IC}_{50} \pm \text { SEM }(\mu \mathrm{M})\right)}$ \\
1 & $0.41 \pm 0.06$ \\
2 & $0.18 \pm 0.01$ \\
3 & $0.46 \pm 0.03$ \\
4 & $1.18 \pm 0.11$ \\
5 & $1.61 \pm 0.14$ \\
Quercetin & $15.8 \pm 0.61$ \\
\hline
\end{tabular}


Molecular docking studies of 15-LOX: To anticipate the binding interactions of most potent inhibitors, molecular docking studies were done for selected compounds 1, 2 and 3 along with cognate ligand (Trans-12,13-epoxy)-9-hydroxy-10(E)-octadecenoic acid) in its respective binding site (PDB ID: 1IK3). The interactions of cognate ligand include hydrogen bonding with Arg726 and His513, while $\pi-\pi$ interactions and $\pi-\sigma$ interactions with Ile772, Leu277, Leu560, Ser510 and Ile857. However, 1 showed significant interactions with Tyr512, Leu376, Arg378 and Pro432 within the active pocket of 15LOX. However, 2 exhibited several interactions like $\pi$ $\pi$ and $\pi-\sigma$ with amino acids Leu376, Pro432, Tyr512, Asn521, Arg378 and Asn375. Most notable interactions were demonstrated by 3 within the active pocket of 15-LOX (Figure 6).

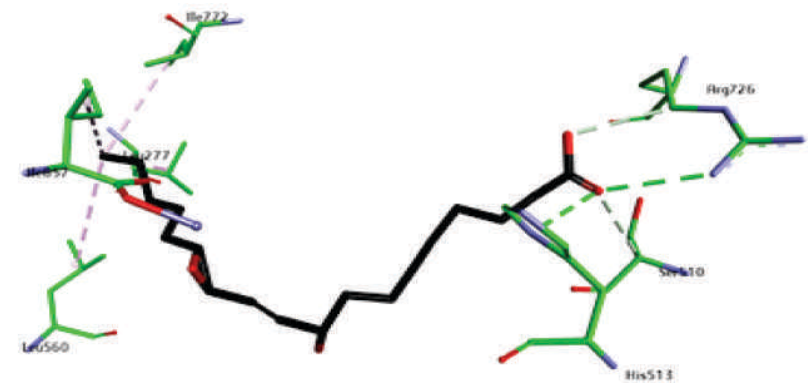

(a)

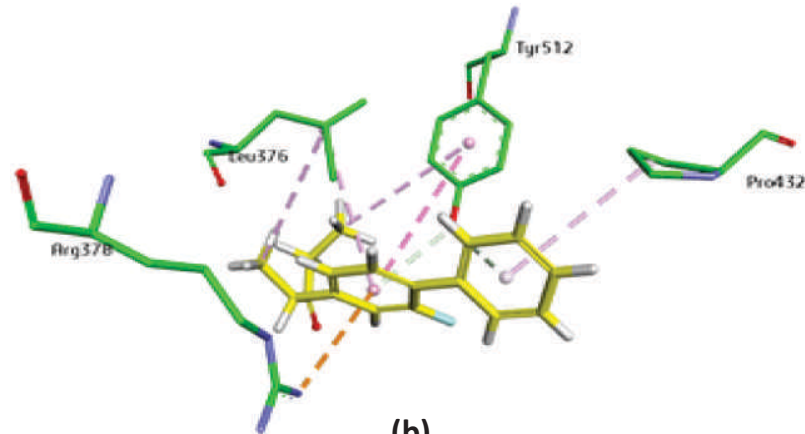

(b)

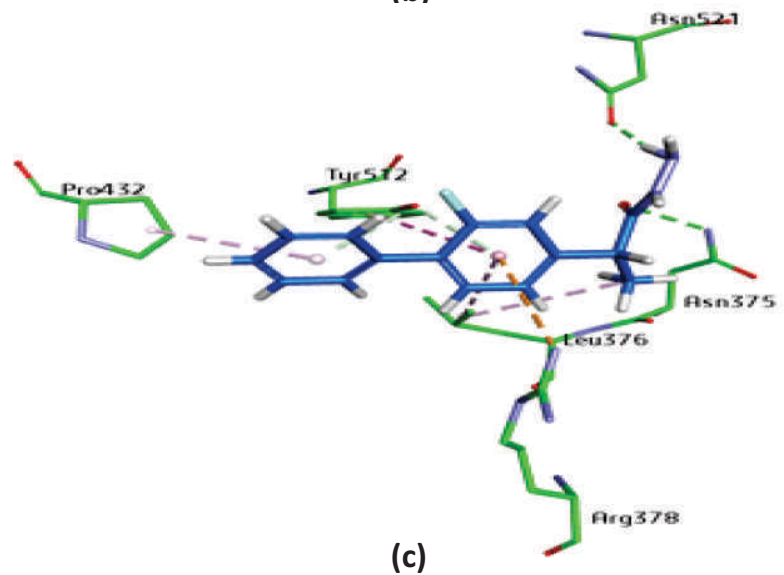

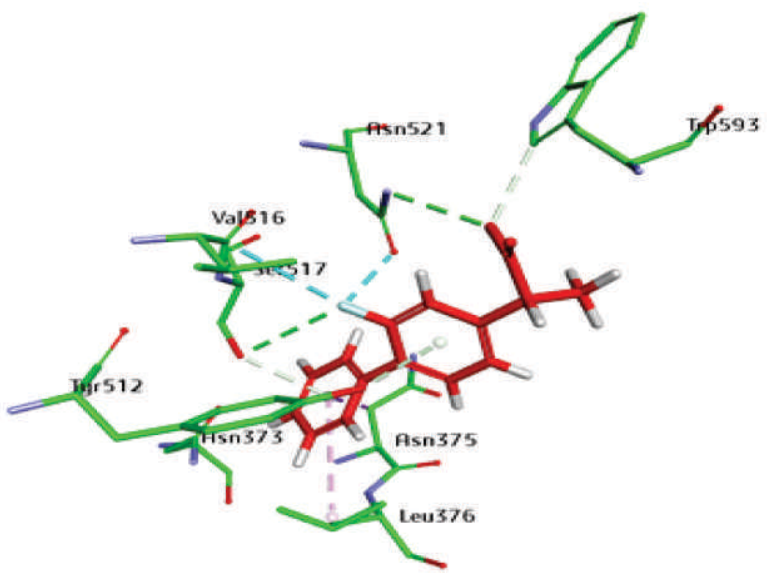

(d)

Fig 6: Docking results of 1, 2, 3 and cognate ligand inside the active site of 15-LOX (PDB ID: 1IK3)

\section{Discussion}

Herein, biologically important ester, hydrazide, thiocarbamate and 1,2,4-trizole-3-thione derivatives of flurbiprofen, (2-(2-fluorobiphenyl-4-yl) propanoic acid) have been synthesized and characterized. The infrared spectroscopic studies indicated that a thione tautomer of 5 is formed which was also confirmed by single crystal X-ray diffraction analysis. In addition to this, the formation of a thione moiety instead of a thiol functional group has been further confirmed by the appearance of a peak at $166.8 \mathrm{ppm}$ for $\{C=S\}$ in ${ }^{13} \mathrm{C}$ NMR spectroscopy. Biological evaluation data shows that compound 2 is the most potent 15-LOX inhibitor with an $\mathrm{IC}_{50}$ value of $0.18 \pm$ $0.01 \mu \mathrm{M}$. Molecular docking results show that 1, 2, 3 and cognate ligand inside the active site of 15-LOX (PDB ID: 1IK3) revealed significant correlation. It is found that 1, 2 and 3 along with cognate ligand in its respective binding site (PDB ID: 1IK3) demonstrated hydrogen bonding, $\pi-\pi$ and $\pi-\sigma$ interactions while 1 exhibited fascinating interactions with Tyr512, Leu376, Arg378 and Pro432 within the active pocket of 15-LOX. Besides, compound 3 demonstrated most significant interactions within the active pocket of 15-LOX., which revealed hydrogen bonding along with $\pi-\pi$ and $\pi-\sigma$ interactions.

\section{Conclusion}

This work represents cost-effective, reproducible, and facile conversion of an aromatic monocarboxylic acid into potent derivatives. This study showed that flurbiprofen (1) (non-selective LOX inhibitor) and its derivatives causes inhibition of LOX enzyme. This is referred as drug repurposing and polypharmacology that result in therapeutic synergism. Inhibition of lipoxygenase causes bronchodilation by reducing leukotrienes. In COPD, inhibition of the enzyme is 
required to reduce pleural effusion and induce bronchodilation. Current study showed that the cognate ligand (Trans-12,13-epoxy)-9-hydroxy10(E)-octadecenoic acid) bound through hydrogen bonding with Arg726 and His513, while it showed $\pi$ $\pi$ interactions and $\pi-\sigma$ interactions with 1 le 772 , Leu277, Leu560, Ser510 and Ile857. 15-LOX inhibition results indicated that the most potent inhibitor (2) for 15-LOX has an $\mathrm{IC}_{50}$ value of $0.18 \pm 0.01$ $\mu \mathrm{M}$. It is concluded that 2-(2-fluorobiphenyl-4-yl) propanoic acid (1) and its derivatives (2-5) possess 15-LOX inhibition and can be a prospective therapeutic target for COPD and atherosclerosis.

\section{Acknowledgements}

J. I. is thankful to the Higher Education Commission of Pakistan for the financial support through Project No. Ph-V-MG-3/Peridot/R\&D/HEC/2019.

\section{REFERENCES}

1. Li QQ, Li Q, Jia JN, Liu ZQ, Zhou HH, Mao XY. Neurochem. Int. 2018; 118: 34-41.

2. Eleftheriadis N, Neochoritis CG, Leus NGJ, van der Wouden PE, Domling A, Dekker FJ. J. Med. Chem. 2015; 58: 7850-62.

3. van der Vlag R, Guo H, Hapko U, Eleftheriadis N, Monjas L, Dekker FJ, et al. Eur. J. Med. Chem. 2019; 174: 45-55.

4. Hu C, Ma S. Med. Chem. Comm. 2018; 9: 212-22.

5. Kuhn H, Banthiya S, Van Leyen K. Biochim. Biophys. Acta Mol. Cell Biol. Lipids. 2015; 1851: 308-30.

6. Brash AR, Lipoxygenases J. Biol. Chem. 1999; 274: 23679-82.

7. Solomon El, Zhou J, Neese F, Pavel EG. Chem. Biol. 1997; 4: 795-808.

8. Brash AR. J. Biol. Chem. 1999; 274: 23679-82.

9. Kuhn H, Thiele BJ. FEBS Lett. 1999; 449: 7-11.

10. Wang MT, Honn KV, Nie D. Cancer Metastasis Rev. 2007; 26: 525-34.

11. Kuhn H, Walther W, Kuban RJ. Prostaglandins Other Lipid Mediat. 2002; 68: 263-90.

12. Pooryaghoobi N, Bakavoli M, Alimardani M, Bazzazan T, Sadeghian H. Iran. J. Basic Med. Sci. 2013; 16: 784-9.

13. Setty B, Werner M, Hannun Y, Stuart M. Blood. 1992; 80: 2565-73.

14. Sultana C, Shen Y, Rattan V, Kalra K. J. Cell. Physiol. 1996; 167: 477-87.

15. Abe M, Yoshimoto T, Zasshi NY. 2004; 124: 415-25.
16. Volovitz B, Welliver RC, De Castro G, Krystofik DA, Ogra PL. Pediatr. Res. 1988; 24: 504-7.

17. Gentile DL, Fireman, Skoner DP. Ann. Allergy. Asthma Immunol. 2003; 91: 270-4.

18. Funk CD. Nat. Rev. Drug Discov. 2005; 4:664-72.

19. Klein $M$, Krainz K, Redwan IN, Dinér $P$, Grøtli $M$. Molecules. 2009; 14: 5124-43.

20. Bayrak H, Demirbas A, Demirbas N, Karaoglu SA. Eur. J. Med. Chem. 2009; 44: 4362-6.

21. El T, Ali S, Mohammed A, Kazak E. Eur. J. Chem. 2010; 1: 7-12.

22. Tehranchian $\mathrm{S}, \mathrm{Akbarzadeh} \mathrm{T}$, Fazeli $\mathrm{MR}$, Jamalifar $\mathrm{H}$, Shafiee A. Bioorg. Med. Chem. Lett. 2005; 15: 1023-5.

23. Papadopoulou MV, Bloomer WD, Rosenzweig HS, Chatelain E, Kaiser M, Wilkinson SR, et al. J. Med. Chem. 2012; 55: 5554-65.

24. Küçükgüzel SG, Küçükgüzel I, Tatar E, Rollas $S$, Şahin $F$, Güllüce M, et al. J. Med. Chem. 2007; 42: 893-901.

25. Amir M, Kumar H, Javed SA, Bioorg. Med. Chem. Lett. 2007; 17:4504-8.

26. Malterud KE, Rydland KM. J. Agric. Food Chem. 2000; 48: 5576-80.

27. Holmes RR, Schmid CG, Chandrasekhar V, Day R, Holmes JM. J. Am. Chem. Soc. 1987; 109: 1408-14.

28. Abbas S, Zaib S, Rahman S, Ali S, Hameed S, Tahir MN, et al. J. Iqbal, Med. Chem. 2019; 15: 298-310.

29. Sheldrick GM. SHELXS-97, Program for X-ray Crystal Structure Solution, Göttingen University, Göttingen, 1997.

30. Sheldrick GM. SHELXS-97, Program for X-ray Crystal Structure Refinement, Göttingen University, Göttingen, 1997.

31. Mustafa G, Khan IU, Ashraf M, Afzal I, Shahzad SA, Shafiq M. Bioorg. Med. Chem. 2012; 20: 2535-39.

32. Morris G, Huey R. J. Comput. Chem. 2009; 30: 2785-91. Chemical Computing Group's Molecular Operating Environment (MOE) MOE 2019.0201. http://www.chemcomp.com/MOEMolecular_Operating_ Environment.htm.

33. LeadIT version 2.3.2; BioSolvelT GmbH, Sankt Augustin, Germany, 2017, www.biosolveit.de/LeadIT.

34. Pettersen EF, Goddard TD, Huang CC, Couch GS, Greenblatt DM, Meng EC, et al. J. Comput. Chem. 2004; 25: 1605-12.

35. BIOVIA Discovery Studio Client v19.1.0.18287. Accelrys Discovery Studio. Accelrys Software Inc, San Diego, 2019.

36. Shaojie Wu, Wenhui Zhang, Le Qi, Yinghui Ren, Haixia Ma. J Mol. Struc. 2019; 5: 171-82. 